\title{
Codimension-m Bifurcation Theorems Applicable to the Numerical Verification Methods
}

\author{
Tadashi Kawanago \\ Department of Mathematics, Tokyo Institute of Technology, 2-12-1 Ookayama, Meguro-ku, \\ Tokyo 152-8551, Japan \\ Correspondence should be addressed to Tadashi Kawanago; tadashi@math.titech.ac.jp
}

Received 20 December 2012; Revised 13 April 2013; Accepted 18 April 2013

Academic Editor: William J. Layton

Copyright (C) 2013 Tadashi Kawanago. This is an open access article distributed under the Creative Commons Attribution License, which permits unrestricted use, distribution, and reproduction in any medium, provided the original work is properly cited.

We establish codimension- $m$ bifurcation theorems applicable to the numerical verification methods. They are generalization of codimension-1 bifurcation theorems established by (Kawanago, 2004). As a numerical example, we treat Hopf bifurcation, which is codimension-2 bifurcation.

\section{Introduction}

By the recent growth of the computer power, we can observe numerically bifurcation phenomena of solutions without difficulty for a lot of differential equations and systems. It is in general difficult, however, to analyze rigorously such phenomena by the use of pure analytical methods. Actually, it seems impossible at least at present to analyze by the use of pure analytical methods the Hopf bifurcation phenomena in the Brusselator model treated in Section 4. We need some computer-assisted analysis to treat it. We now have various excellent bifurcation theorems from the theoretical point of view. It needs in general, however, some particular devices to apply them to a given concrete dynamical system since we are usually not able to check some conditions in such theorems directly by numerical methods.

Another important approach to computer-assisted analysis for bifurcation problems is to establish new bifurcation theorems applicable directly to numerical verification methods. This approach is our theme in this paper. It is useful from the practical and applied mathematical point of view. In [1], the author established some codimension-1 bifurcation theorems applicable directly to numerical verification methods. Using a symmetry-breaking bifurcation theorem $[1$, Theorem 3.1] and the numerical verification methods, we proved the existence of a $\mathbb{Z}_{2}$-symmetry-breaking bifurcation point for a nonlinear forced vibration system described by a wave equation in [2], and Nakao et al. verified some symmetrybreaking bifurcation points for two-dimensional RayleighBénard heat convection system in $[3,4]$.

In this paper, we establish codimension- $m$ bifurcation theorems applicable to the numerical verification methods. They are generalization of codimension-1 bifurcation theorems mentioned above. In Section 4, we apply our new theorem to Hopf bifurcation, which is codimension-2 bifurcation.

Here, we present our main theorem. Let $X$ and $Y$ be real Banach spaces. Let $X_{1}$ and $X_{2}$ be closed subspaces of $X$ and let $Y_{1}$ and $Y_{2}$ be closed subspaces of $Y$. We assume that $X=X_{1} \oplus X_{2}$ and $Y=Y_{1} \oplus Y_{2}$. Here, $\oplus$ means the direct sum. Let $m \in \mathbb{N}$ and $g \in C^{2}\left(\mathbb{R}^{m} \times X, Y\right)$ have the following properties:

$$
\begin{gathered}
\qquad g(\Lambda, v) \in Y_{1} \quad \text { for any } \Lambda \in \mathbb{R}^{m}, v \in X_{1}, \\
g_{u}(\Lambda, v) X_{j} \subset Y_{j} \quad \text { for any } \Lambda \in \mathbb{R}^{m}, v \in X_{1}(j=1,2), \\
g_{u u}\left(\Lambda, v_{1}\right) v_{2} w \in Y_{2} \text { for any } \Lambda \in \mathbb{R}^{m}, v_{1}, v_{2} \in X_{1}, w \in X_{2} .
\end{gathered}
$$


We denote by $\mathbf{e}_{1}:=(1,0, \ldots, 0) \in \mathbb{R}^{m}$ the first row vector of the identity matrix of order $m$. We define $J: \mathbb{R}^{m} \times X \rightarrow \mathbb{R}^{m} \times Y$ by

$$
\begin{aligned}
J\left(\begin{array}{c}
\Lambda \\
v \\
w
\end{array}\right):= & \left(\begin{array}{c}
l w-\mathbf{e}_{1} \\
g(\Lambda, v) \\
g_{u}(\Lambda, v) w
\end{array}\right) \in \mathbb{R}^{m} \times Y_{1} \times Y_{2} \\
& \text { for }(\Lambda, v, w) \in \mathbb{R}^{m} \times X_{1} \times X_{2} .
\end{aligned}
$$

Here, $l \in \mathscr{L}\left(X, \mathbb{R}^{m}\right)$, and we assume that $l v=0$ for any $v \in X_{1}$. We define projections $p$ and $P$ by

$$
\begin{gathered}
p:\left(r_{1}, r_{2}, \ldots, r_{m}\right) \in \mathbb{R}^{m} \longmapsto r_{1} \in \mathbb{R}, \\
P:\left(r_{1}, r_{2}, \ldots, r_{m}\right) \in \mathbb{R}^{m} \longmapsto\left(r_{2}, \ldots, r_{m}\right) \in \mathbb{R}^{m-1}
\end{gathered}
$$

for the case $m \geq 2$.

In what follows, we always set formally $\mathbb{R}^{m-1} \times Y:=Y$ for the case: $m=1$. We define $G: \mathbb{R}^{m} \times X \rightarrow \mathbb{R}^{m-1} \times Y$ by

$$
\begin{aligned}
G & :=g \quad(m=1) \\
G\left(\begin{array}{l}
\Lambda \\
u
\end{array}\right) & :=\left(\begin{array}{c}
P l u \\
g(\Lambda, u)
\end{array}\right) \quad(m \geq 2) .
\end{aligned}
$$

We set $Z:=(p l)^{-1}(0)=\{u \in X$; plu $=0\}$ and $\mathbb{R}_{+}:=$ $(0, \infty)$. Our main theorem is the following.

Theorem 1. In addition to the assumptions above, we assume that $\left(\Lambda_{0}, v_{0}, w_{0}\right) \in \mathbb{R}^{m} \times X_{1} \times X_{2}$ satisfies the following (H1) and $(\mathrm{H} 2)$ :

(H1) A point $(\Lambda, v, w)=\left(\Lambda_{0}, v_{0}, w_{0}\right)$ is an isolated solution of the extended system $J(\Lambda, v, w)=0$.

(H2) The linear operator $\left.g_{u}\left(\Lambda_{0}, v_{0}\right)\right|_{X_{1}}: X_{1} \rightarrow Y_{1}$ is bijective.

Then, the point $\left(\Lambda_{0}, v_{0}\right)$ is a bifurcation point of the equation $G(\Lambda, v)=0$. Exactly, there exist an open neighborhood $W$ of $\left(\Lambda_{0}, v_{0}\right)$ in $\mathbb{R}^{m} \times X, a \in \mathbb{R}_{+}, \zeta \in C^{1}\left((-a, a), \mathbb{R}^{m}\right), \eta \in$ $C^{1}((-a, a), Z)$, an open neighborhood $V$ of 0 in $\mathbb{R}^{m}$ and $q \in$ $C^{2}\left(\Lambda_{0}+V, X_{1}\right)$ such that $\zeta(0)=\Lambda_{0}, \eta(0)=0, q\left(\Lambda_{0}\right)=v_{0}$ and

$$
\begin{aligned}
G^{-1}(0) \cap W= & \{(\Lambda, q(\Lambda)) ;(\Lambda, q(\Lambda)) \in W\} \\
& \cup\left\{\left(\zeta(\alpha), \alpha w_{0}+\alpha \eta(\alpha)+q(\zeta(\alpha))\right) ;|\alpha|<a\right\} .
\end{aligned}
$$

Roughly speaking, the well-known pitchfork bifurcation theorem [5, Theorem 1.7] by Crandall and Rabinowitz is equivalent to Theorem 1 with $m=1, X_{1}=\{0\}$, and $Y_{1}=$ $\{0\}$ (see Section 2). We immediately obtain a $\mathbb{Z}_{2}$-symmetry breaking bifurcation theorem [1, Theorem 3.1] by setting $m=$ 1 in Theorem 1 and by choosing the symmetric subspace of $X$ as $X_{1}$ and the anti-symmetric subspace as $X_{2}$. We can apply Theorem 1 to Hopf bifurcation by setting $m=2$ and choosing an appropriate space of periodic functions as $X$, the subspace of steady functions as $X_{1}$, and the complementary subspace of $X_{1}$ as $X_{2}$ (see Section 4 below).

Finally, we illustrate by Hopf bifurcation as an example that known bifurcation theorems are not in general applicable directly to the numerical verification methods. We consider the next autonomous ordinary differential equation:

$$
\dot{y}=f(\lambda, y), \quad y, f(\lambda, y) \in \mathbb{R}^{d} .
$$

The classical Hopf bifurcation theorem reads as follows.

Theorem 2. The point $(\lambda, y)=\left(\lambda_{0}, y_{0}\right)$ is a Hopf bifurcation point of $(O)$, that is, a branch of periodic solutions of $(O)$ bifurcates at the point $(\lambda, y)=\left(\lambda_{0}, y_{0}\right)$ from a branch of steady solutions of $(O)$ with the initial period $2 \pi \sigma_{0}$, provided that the following conditions (C1)-(C4) are satisfied:

(C1) $f\left(\lambda_{0}, y_{0}\right)=0$,

(C2) $\pm i$ are the simple eigenvalues of $\sigma_{0} f_{y}\left(\lambda_{0}, y_{0}\right)$ (so, by the implicit function theorem, the matrix $\sigma_{0} f_{y}(\lambda, y(\lambda))$ has a pair of complex conjugate of eigenvalues $\mu(\lambda)$, $\overline{\mu(\lambda)}$ with $\left.\mu\left(\lambda_{0}\right)=i\right)$,

(C3) (Transversality condition of eigenvalues) $\operatorname{Re} \mu^{\prime}\left(\lambda_{0}\right) \neq 0$,

(C4) ik is not an eigenvalue of $\sigma_{0} f_{y}\left(\lambda_{0}, y_{0}\right)$ for $k \in \mathbb{Z}-$ $\{-1,1\}$.

It is difficult to check rigorously by numerical methods the dynamic condition (C3), and we need a particular device for it. See Remark 15 for more detailed information. On the other hand, the condition (C3) is implied by (H1) and (H2) in Theorem 1, which are static conditions, that is, regularity conditions for linear operators.

The paper is organized as follows. In Section 2, we mainly establish a basic bifurcation theorem, which is simpler than Theorem 1. In Section 3, we prove Theorem 1. In Section 4, we present a numerical example and treat Hopf bifurcation as an application of Theorem 1 with $m=2$ and our numerical verification method. We present some final remarks in Section 5.

Notation. Let $\mathscr{H}$ and $\mathscr{K}$ be Banach spaces.

(1) We denote by $\mathbb{R}$ the set of all real numbers, by $\mathbb{Q}$ the set of all rational numbers, by $\mathbb{Z}$ the set of all integers, and by $\mathbb{C}$ the set of all complex numbers. We write $\mathbb{R}_{+}:=(0, \infty)$.

(2) We denote by $\mathbf{e}_{1}:=(1,0, \ldots, 0) \in \mathbb{R}^{m}$ the first row vector of the identity matrix $E_{m}=\left(\delta_{i j}\right)$ of order $m$.

(3) We denote by $\|\cdot\|_{\mathscr{H}}$ the norm of $\mathscr{H}$. If $\mathscr{H}$ is a Hilbert space, then $\|\cdot\|_{\mathscr{H}}$ stands for the norm induced by the inner product of $\mathscr{H}$. For $u \in \mathscr{H}$ and $r \in(0, \infty)$, we write $B_{\mathscr{H}}(u ; r):=\{v \in \mathscr{H} ;\|v-u\|<r\}$. The subscript will be often omitted if no possible confusion arises.

(4) We denote by $\mathscr{L}(\mathscr{H}, \mathscr{K})$ the space of bounded linear operators from $\mathscr{H}$ to $\mathscr{K}$, and $\mathscr{L}(\mathscr{H})$ stands for $\mathscr{L}(\mathscr{H}, \mathscr{H})$. For $T \in \mathscr{L}(\mathscr{H}, \mathscr{K}),\|T\|_{\mathscr{H} \rightarrow \mathscr{K}}$ denotes the operator norm of $T$. The subscript will be omitted if no possible confusion arises. 
(5) Let $A: \mathscr{H} \rightarrow \mathscr{K}$ be an operator. We denote by $\mathscr{D}(A)$ the domain of $A$ and by $\mathscr{R}(A)$ the range of $A$. We define $\mathcal{N}(A):=\{v \in \mathscr{D}(A) ; A v=0\}$.

(6) For an open set $V \subset \mathscr{H}$, we denote by $C^{n}(V, \mathscr{K})$ the space of $n$-times continuously differentiable functions from $V$ to $\mathscr{K}$.

(7) Let $\Omega$ be a domain of $\mathbb{R}^{n}$. We denote Lebesgue spaces by $L^{p}(\Omega)(1 \leq p \leq \infty)$ with the norms: $\|u\|_{L^{p}(\Omega)}=$ $\left(\int_{\Omega}|u(x)|^{p} d x\right)^{1 / p}$ for $1 \leq p<\infty,\|u\|_{L^{\infty}(\Omega)}=$ ess. $\sup \{|u(x)| ; x \in \Omega\}$. We define the norm of the Sobolev space $H^{1}(\Omega)$ by

$$
\|u\|_{H^{1}(\Omega)}:=\left(\|u\|_{L^{2}(\Omega)}^{2}+\sum_{k=1}^{n}\left\|\frac{\partial u}{\partial x_{k}}\right\|_{L^{2}(\Omega)}^{2}\right)^{1 / 2} .
$$

(8) Let $N \in \mathbb{N}$ and $Z_{k}(k=1,2, \ldots, N)$ be norm spaces. We denote their direct product space $Z$ usually by $Z=Z_{1} \times \cdots \times Z_{N}$ and also by $Z=Z_{1} \oplus \cdots \oplus Z_{N}$ in Section 4.5 below. Actually, the direct product space is interchangeably called the direct sum space (see, e.g., $\left[6\right.$, Section II.1]). We define $\|u\|_{Z}:=\left(\sum_{k=1}^{N}\left\|u_{k}\right\|_{Z_{k}}^{2}\right)^{1 / 2}$ for $u=\left(u_{1}, \ldots, u_{N}\right) \in Z_{1} \times \cdots \times Z_{N}$.

\section{Basic Bifurcation Theorems}

Theorem 3 in this section is a generalized version of [1, Theorem 2.1] and can be regarded as a refined version of Theorem 1 with $X_{1}=\{0\}$ and $Y_{1}=\{0\}$.

Let $X$ and $Y$ be real Banach spaces and $U$ be an open neighborhood of 0 in $X$. Let $m \in \mathbb{N}$ and $V$ be an open neighborhood of 0 in $\mathbb{R}^{m}$. Let $f \in C^{1}(V \times U, Y)$ be a map such that

$$
f(\Lambda, 0)=0 \quad \text { for any } \Lambda=\left(\Lambda_{1}, \ldots, \Lambda_{m}\right) \in V \text {, }
$$

and the partial Fréchet derivative $f_{\Lambda_{k} u}$ exists and is continuous for $k=1, \ldots, m$. Here, for any fixed $\left(\Lambda_{\star}, u_{\star}\right) \in V \times U$, $f_{\Lambda_{k} u}\left(\Lambda_{\star}, u_{\star}\right)$ represents the Fréchet derivative of the function $u \in U \mapsto\left(\partial f / \partial \Lambda_{k}\right)\left(\Lambda_{\star}, u\right) \in Y$ at $u=u_{\star}$. We denote $f_{\Lambda u}:=\left(f_{\Lambda_{1} u}, \ldots, f_{\Lambda_{m} u}\right)$ for simplicity. We define $H: V \times$ $X \rightarrow \mathbb{R}^{m} \times Y$ by

$$
H\left(\begin{array}{l}
\Lambda \\
u
\end{array}\right):=\left(\begin{array}{c}
l u-e_{1} \\
f_{u}(\Lambda, 0) u
\end{array}\right)
$$

Here, $l \in \mathscr{L}\left(X, \mathbb{R}^{m}\right)$. In what follows, we often use the same notations as in Section 1. We define $F: V \times U \rightarrow \mathbb{R}^{m-1} \times Y$ by

$$
\begin{gathered}
F:=f \quad(m=1), \\
F\left(\begin{array}{l}
\Lambda \\
u
\end{array}\right):=\left(\begin{array}{c}
P l u \\
f(\Lambda, u)
\end{array}\right) \quad(m \geq 2) .
\end{gathered}
$$

We set $Z:=(p l)^{-1}(0)=\{u \in X ; p l u=0\}$.
Theorem 3. In addition to the assumptions above, we assume that

(H) there exists $u_{0} \in U$ such that $(\Lambda, u)=\left(0, u_{0}\right)$ is an isolated solution of the extended system $H(\Lambda, u)=0$.

Then, there exist an open neighborhood $W$ of $(0,0)$ in $\mathbb{R}^{m} \times X$, $a \in \mathbb{R}_{+}$and continuous functions $\zeta:(-a, a) \rightarrow \mathbb{R}^{m}, \eta:$ $(-a, a) \rightarrow Z$ such that $\zeta(0)=0, \eta(0)=0$ such that

$$
\begin{aligned}
F^{-1}(0) \cap W= & \{(\Lambda, 0) ;(\Lambda, 0) \in W\} \\
& \cup\left\{\left(\zeta(\alpha), \alpha u_{0}+\alpha \eta(\alpha)\right) ; \alpha \in(-a, a)\right\} .
\end{aligned}
$$

Moreover, if $f \in C^{k+1}(k \in \mathbb{N})$, then $\zeta, \eta \in C^{k}$.

For simplicity, we write $D H^{0}:=D H\left(0, u_{0}\right), f_{u}^{0}:=$ $f_{u}(0,0), F_{u}^{0}:=F_{u}(0,0), F_{\Lambda u}^{0}:=F_{\Lambda u}(0,0)$, and so on. We have

$$
D H^{0}\left(\begin{array}{l}
\Lambda \\
u
\end{array}\right)=\left(\begin{array}{c}
l u \\
\Lambda \cdot f_{\Lambda u}^{0} u_{0}+f_{u}^{0} u
\end{array}\right)=\left(\begin{array}{c}
p l u \\
\Lambda \cdot F_{\Lambda u}^{0} u_{0}+F_{u}^{0} u
\end{array}\right) .
$$

Proof of Theorem 3. The proof is similar to that of [1, Theorem 2.1] and [5, Theorem 1.7]. We obtain from $(\mathrm{H})$ that $p l u_{0}=1$ and $F_{u}^{0} u_{0}=0$. Let $\widehat{u}$ be any element of $\mathcal{N}\left(F_{u}^{0}\right)$. We set $\widehat{c}:=p l \widehat{u}$. Then, we have $D H^{0}\left(0, \widehat{u}-\widehat{c} u_{0}\right)=0$. Since $D H^{0}$ is one to one, $\widehat{u}=\widehat{c} u_{0}$. So we obtain $\mathcal{N}\left(F_{u}^{0}\right)=\operatorname{span}\left\{u_{0}\right\}$. We choose $\varepsilon \in \mathbb{R}_{+}$and an open neighborhood $K$ of 0 in $X$ such that $\alpha\left(u_{0}+u\right) \in U$ for $(\alpha, u) \in(-\varepsilon, \varepsilon) \times K$. We define a map $h:(-\varepsilon, \varepsilon) \times V \times K \rightarrow \mathbb{R}^{m} \times Y$ by

$$
\begin{aligned}
h(\alpha, \Lambda, u):=\left(\begin{array}{c}
l u \\
h_{0}(\alpha, \Lambda, u)
\end{array}\right) \\
\text { with } h_{0}(\alpha, \Lambda, u):= \begin{cases}\frac{1}{\alpha} f\left(\Lambda, \alpha u_{0}+\alpha u\right) & (\alpha \neq 0), \\
f_{u}(\Lambda, 0)\left(u_{0}+u\right) & (\alpha=0) .\end{cases}
\end{aligned}
$$

By $H\left(0, u_{0}\right)=0$, we have $h(0,0,0)=\left(0, f_{u}^{0} u_{0}\right)=0$. We verify that the partial derivative of $h(\alpha, \Lambda, u)$ with respect to $(\Lambda, u)$ at $(\alpha, \Lambda, u)=(0,0,0)$ coincides with $D H^{0}$ and is bijective. So, by applying the implicit function theorem to $h(\alpha, \Lambda, u)$ at $(\alpha, \Lambda, u)=(0,0,0)$, there exist an open neighborhood $W \subset V \times K$ of $(0,0), a \in(0, \varepsilon)$ and continuous functions $\zeta:(-a, a) \rightarrow \mathbb{R}^{m}, \eta:(-a, a) \rightarrow Z$ such that $\zeta(0)=0$, $\eta(0)=0$, and $h(\alpha, \zeta(\alpha), \eta(\alpha))=0$ for any $\alpha \in(-a, a)$ and that $h(\alpha, \Lambda, u)=0$ and $(\alpha, \Lambda, u) \in(-a, a) \times W$ imply $\Lambda=\zeta(\alpha)$ and $u=\eta(\alpha)$. Thus, $F^{-1}(0) \cap W$ includes the right-hand side of (12). From the same argument as in the proof of [5, Theorem 1.7], we can verify that (12) actually holds.

In view of the next result, we may consider Theorem 3 as a generalized version of [5, Theorem 1.7]. 
Proposition 4. The condition $(H)$ in Theorem 3 is equivalent to the following (i) and (ii):

(i) $\operatorname{dim} \mathscr{N}\left(F_{u}^{0}\right)=1$ and $\operatorname{codim} \mathscr{R}\left(F_{u}^{0}\right)=m$,

(ii) $\exists u_{0} \in U$ such that

$$
\begin{gathered}
\operatorname{plu}_{0}=1, \quad \mathcal{N}\left(F_{u}^{0}\right)=\operatorname{span}\left\{u_{0}\right\}, \\
\operatorname{span}\left(F_{\Lambda u}^{0} u_{0}\right) \oplus \mathscr{R}\left(F_{u}^{0}\right)=\mathbb{R}^{m-1} \times Y .
\end{gathered}
$$

Here, we denote $\operatorname{span}\left(F_{\Lambda u}^{0} u_{0}\right):=\left\{\Lambda \cdot F_{\Lambda u}^{0} u_{0} ; \Lambda \in \mathbb{R}^{m}\right\}$.

Proof. First, we assume (H). By the proof of Theorem 3, we have $\operatorname{dim} \mathcal{N}\left(F_{u}^{0}\right)=1$ and (15). Since $D H^{0}$ is onto, we have $\operatorname{span}\left(F_{\Lambda u}^{0} u_{0}\right)+\mathscr{R}\left(F_{u}^{0}\right)=\mathbb{R}^{m-1} \times Y$. Let $p \in \operatorname{span}\left(F_{\Lambda u}^{0} u_{0}\right)$ and $q \in R\left(F_{u}^{0}\right)$ satisfy $p+q=0$. In order to prove (16), it suffices to show $p=q=0$. We choose $\widetilde{\Lambda} \in \mathbb{R}^{m}$ and $\tilde{u} \in X$ such that $p=\widetilde{\Lambda} \cdot F_{\Lambda u}^{0} u_{0}$ and $q=F_{u}^{0} \widetilde{u}$. We set $\widetilde{c}:=$ pl $\tilde{u}$. Then, $D H^{0}\left(\widetilde{\Lambda}, \tilde{u}-\widetilde{c} u_{0}\right)=0$. We have $\widetilde{\Lambda}=0$ and $\tilde{u}-\widetilde{c} u_{0}=0$ since $D H^{0}$ is an injective linear map. So, we obtain $p=0$, which implies $q=0$. Thus, (16) holds. Next, we show by the same discussion as above that $\left\{F_{\Lambda_{k} u}^{0} u_{0}\right\}_{k=1}^{m}$ is linearly independent. Let $\Lambda \in \mathbb{R}^{m}$ satisfy $\Lambda \cdot F_{\Lambda u}^{0} u_{0}=0$. Then, we have $D H^{0}(\Lambda, 0)=0$, which implies $\Lambda=0$. Thus, $\left\{F_{\Lambda_{k} u}^{0} u_{0}\right\}_{k=1}^{m}$ is linearly independent. So, $\operatorname{dim} \operatorname{span}\left(F_{\Lambda u}^{0} u_{0}\right)=m$. By this and (16), we have codim $\mathscr{R}\left(F_{u}^{0}\right)=m$. Thus, (i) and (ii) hold.

We show the inverse. We assume (i) and (ii). Clearly, (15) implies $l u_{0}=\mathbf{e}_{1}$ and $H\left(0, u_{0}\right)=0$. So, it suffices to show that $D H^{0}$ is bijective. Let $(\widehat{\Lambda}, \widehat{u}) \in \mathbb{R}^{m} \times X$ satisfy $D H^{0}(\widehat{\Lambda}, \widehat{u})=0$. Then, by (13) we have pl $\widehat{u}=0$ and $\widehat{\Lambda} \cdot F_{\Lambda u}^{0} u_{0}+F_{u}^{0} \widehat{u}=0$. We obtain from (16) that $\widehat{\Lambda} \cdot F_{\Lambda u}^{0} u_{0}=0$ and $F_{u}^{0} \widehat{u}=0$. We have $\widehat{\Lambda}=0$ since (i) and (16) imply $\operatorname{span}\left(F_{\Lambda u}^{0} u_{0}\right)=m$. By pl $\widehat{u}=0$ and (15), we have $\widehat{u}=0$. Hence, $D H^{0}$ is one to one. Finally, let $(\lambda, y)$ be an element of $\mathbb{R}^{m} \times Y$. By (16), there exist $\widetilde{\Lambda} \in \mathbb{R}^{m}$ and $\tilde{u} \in X$ such that $\widetilde{\Lambda} \cdot F_{\Lambda u}^{0} u_{0}+F_{u}^{0} \widetilde{u}=(P \lambda, y)$. We set $\widetilde{c}:=p(\lambda-l \widetilde{u})$. Then, $D H^{0}\left(\widetilde{\Lambda}, \widetilde{u}+\widetilde{c} u_{0}\right)=(\lambda, y)$. Thus, $D H^{0}$ is onto. The proof is complete.

\section{Proof of Theorem 1}

We set $D J^{0}:=D J\left(\Lambda_{0}, v_{0}, w_{0}\right)$. We use the same notations as used in Section 2. We set $g_{u}^{0}:=g_{u}\left(\Lambda_{0}, v_{0}\right), g_{\Lambda}^{0}:=g_{\Lambda}\left(\Lambda_{0}, v_{0}\right)$, $g_{\Lambda u}^{0}:=g_{\Lambda u}\left(\Lambda_{0}, v_{0}\right)$, and $g_{u u}^{0}:=g_{u u}\left(\Lambda_{0}, v_{0}\right)$. Then, we have

$$
D J^{0}\left(\begin{array}{c}
\Lambda \\
v \\
w
\end{array}\right)=\left(\begin{array}{c}
l w \\
\Lambda \cdot g_{\Lambda}^{0}+g_{u}^{0} v \\
\Lambda \cdot g_{\Lambda u}^{0} w_{0}+g_{u u}^{0} v w_{0}+g_{u}^{0} w
\end{array}\right) \text {. }
$$

Proof of Theorem 1. By (H2) and the implicit function theorem, there exist an open neighborhood $V$ of 0 in $\mathbb{R}^{m}$ and a map $q=q(\Lambda) \in C^{2}\left(\Lambda_{0}+V, X_{1}\right)$ such that $q\left(\Lambda_{0}\right)=v_{0}$ and $g(\Lambda, q(\Lambda))=0$ for any $\Lambda \in \Lambda_{0}+V$. We define $f: V \times X \rightarrow Y$ by

$$
f(\Lambda, u):=g\left(\Lambda_{0}+\Lambda, u+q\left(\Lambda_{0}+\Lambda\right)\right) .
$$

Then, we have (9), $f_{u}^{0}=g_{u}^{0}$, and

$$
f_{\Lambda u}^{0} w_{0}=g_{\Lambda u}^{0} w_{0}+g_{u u}^{0} \mathbf{x}_{0} w_{0} \quad \text { with } \mathbf{x}_{0}:=q^{\prime}\left(\Lambda_{0}\right) \in X_{1}^{m}
$$

It follows from (9) and ( $\mathrm{H} 2)$ that $\mathbf{x}_{0} \in X_{1}^{m}$ is uniquely determined by the equation:

$$
\left(f_{\Lambda}(0,0)\right)=g_{\Lambda}^{0}+g_{u}^{0} \mathbf{x}_{0}=0
$$

We define $H: V \times X \rightarrow \mathbb{R}^{m} \times Y$ by (10), and set $u_{0}:=$ $w_{0}$. Then, we have (13). In view of Theorem 3 , the proof is complete if we show that the map $D H^{0}: \mathbb{R}^{m} \times X \rightarrow \mathbb{R}^{m} \times Y$ is bijective. Let $(\Lambda, u) \in \mathbb{R}^{m} \times X$ satisfy $D H^{0}(\Lambda, u)=0$. It follows from (2), (3), (13), and (19) that

$$
\begin{gathered}
l u=0, \\
g_{u}^{0} u=f_{u}^{0} u=-\Lambda \cdot f_{\Lambda u}^{0} w_{0}=-\Lambda \cdot\left(g_{\Lambda u}^{0} w_{0}+g_{u u}^{0} \mathbf{x}_{0} w_{0}\right) \in Y_{2} .
\end{gathered}
$$

So, by (2), (H2), (17), (20), and (21), we have $u \in X_{2}$ and $D J^{0}\left(\Lambda, \Lambda \cdot \mathbf{x}_{0}, u\right)=0$. We obtain $(\Lambda, u)=0$ since $D J^{0}$ is one to one. Hence, $D H^{0}$ is one to one. Next, let $(\lambda, y)$ be an element of $\mathbb{R}^{m} \times Y$. Let $y=y_{1}+y_{2}$ with $y_{1} \in Y_{1}$ and $y_{2} \in Y_{2}$. Since $D J^{0}$ is onto, there exists $(\widetilde{\Lambda}, \widetilde{v}, \widetilde{w}) \in \mathbb{R}^{m} \times X_{1} \times X_{2}$ such that $\operatorname{DJ}^{0}(\widetilde{\Lambda}, \widetilde{v}, \widetilde{w})=\left(\lambda, 0, y_{2}\right)$. By (H2), (17), (19), and (20), we have $\lambda=l \widetilde{w}, \widetilde{v}=\widetilde{\Lambda} \cdot \mathbf{x}_{0}$, and $y_{2}=\widetilde{\Lambda} \cdot\left(g_{\Lambda u}^{0} w_{0}+g_{u u}^{0} \mathbf{x}_{0} w_{0}\right)+g_{u}^{0} \widetilde{w}=$ $\widetilde{\Lambda} \cdot f_{\Lambda u}^{0} w_{0}+f_{u}^{0} \widetilde{w}$. We set $\tilde{u}:=\left(\left.g_{u}^{0}\right|_{X_{1}}\right)^{-1} y_{1} \in X_{1}$. Then, by (19), we have $D H^{0}(\widetilde{\Lambda}, \widetilde{u}+\widetilde{w})=(\lambda, y)$. Therefore, $D H^{0}$ is onto. The proof is complete.

\section{A Numerical Example}

In this section, we present a numerical example. We treat Hopf bifurcation as an application of Theorem 1 with $m=2$. This section is organized as follows. In Section 4.1, we present a partial differential system we consider. In Section 4.2, we rewrite our problem under appropriate setting of functional spaces. In Section 4.3, we describe our numerical verification result on the existence of a Hopf bifurcation point. In Section 4.4, we present the principal abstract results in our numerical verification methods. In Section 4.5, we describe the outline of derivation of our verification result. 
4.1. Brusselator Model. We consider the following Brusselator model:

$$
\begin{aligned}
& \frac{\partial u_{1}}{\partial t}-\frac{\partial^{2} u_{1}}{\partial x^{2}}=(A+\eta \cos x)-(B+1) u_{1} \\
& +u_{1}^{2} u_{2}+\lambda\left(u_{3}-u_{1}\right) \\
& \text { for }(x, t) \in(0, \pi) \times[0, \infty) \text {, } \\
& \frac{\partial u_{2}}{\partial t}-\frac{\partial^{2} u_{2}}{\partial x^{2}}=B u_{1}-u_{1}^{2} u_{2}+\frac{\lambda}{\rho}\left(u_{4}-u_{2}\right) \\
& \text { for }(x, t) \in(0, \pi) \times[0, \infty) \text {, } \\
& \frac{\partial u_{3}}{\partial t}-\frac{\partial^{2} u_{3}}{\partial x^{2}}=(A+\eta \cos x)-(B+1) u_{3} \\
& +u_{3}^{2} u_{4}+\lambda\left(u_{1}-u_{3}\right) \\
& \text { for }(x, t) \in(0, \pi) \times[0, \infty) \text {, } \\
& \frac{\partial u_{4}}{\partial t}-\frac{\partial^{2} u_{4}}{\partial x^{2}}=B u_{3}-u_{3}^{2} u_{4}+\frac{\lambda}{\rho}\left(u_{2}-u_{4}\right) \\
& \text { for }(x, t) \in(0, \pi) \times[0, \infty) \text {, } \\
& \frac{\partial u_{j}}{\partial x}(0, t)=\frac{\partial u_{j}}{\partial x}(\pi, t)=0 \\
& \text { for } t \in[0, \infty), j=1,2,3,4 \text {. }
\end{aligned}
$$

Here, $A, B, \rho, \lambda$, and $\eta$ are parameters of the problem. In what follows, we set $A:=2, B:=5.9, \rho:=0.1$, and $\eta:=0.001$ and consider $\lambda$ as the bifurcation parameter. By some numerical methods, Kubíček and Holodniok [7] found some period-doubling bifurcation points for an ordinary differential system related closely to (B). On the other hand, we verified the existence of a Hopf bifurcation point for (B).

We set $u=\left(u_{1}, u_{2}, u_{3}, u_{4}\right)$. We denote the equations of (B) by $u_{t}-u_{x x}=h(\lambda, u)$. We also write $u_{t}=\varphi(\lambda, u)$ with $\varphi(\lambda, u):=u_{x x}+h(\lambda, u)$. Clearly, $u$ is a $2 \pi \sigma$-periodic solution of (B) if and only if $u$ is a $2 \pi$-periodic solution of the next equation:

$$
u_{t}=\sigma \varphi(\lambda, u)
$$

4.2. The Setting of Functional Spaces. Let $D:=(0, \pi) \times(0,2 \pi)$, $\mathcal{Y}:=L^{2}(D)$, and $Y:=\mathscr{Y}^{4}$. We define a real Hilbert space $\mathscr{X}$ by

$$
\begin{aligned}
\mathscr{X}:= & \left\{u=\sum_{k=-\infty}^{\infty} \sum_{n=0}^{\infty} c_{k n} e^{i k t} \cos n x \in \mathcal{Y} ; c_{k n} \in \mathbb{C}, c_{-k, n}=\overline{c_{k n}}\right. \\
& \text { for any } \left.k \in \mathbb{Z}, n \in \mathbb{N} \cup\{0\} \text { and } u_{t}-u_{x x} \in \mathcal{Y}\right\} \\
= & \left\{\sum_{k=-\infty}^{\infty} \sum_{n=0}^{\infty} c_{k n} e^{i k t} \cos n x \in \mathscr{Y} ; c_{k n} \in \mathbb{C}, c_{-k, n}=\overline{c_{k n}}\right.
\end{aligned}
$$

for any $k \in \mathbb{Z}, n \in \mathbb{N} \cup\{0\}$ and

$$
\left.\sum_{k=0}^{\infty} \sum_{n=0}^{\infty}\left(k^{2}+n^{4}\right)\left|c_{k n}\right|^{2}<\infty\right\},
$$

with the inner product

$$
\begin{aligned}
(u, v)_{X}:= & \left(u_{t}-u_{x x}+\frac{1}{2 \pi^{2}} \int_{0}^{2 \pi} d t \int_{0}^{\pi} u d x,\right. \\
& \left.v_{t}-v_{x x}+\frac{1}{2 \pi^{2}} \int_{0}^{2 \pi} d t \int_{0}^{\pi} v d x\right)_{y}
\end{aligned}
$$

for $u, v \in \mathscr{X}$. Then, we easily verify that $\mathscr{X}$ is continuously embedded in $H^{1}(D)$ and that

$$
\begin{aligned}
\|u\|_{\mathscr{X}}= & \sqrt{(u, u)_{X}} \\
= & \pi\left\{2\left|c_{00}\right|^{2}+2 \sum_{k=-\infty}^{\infty} k^{2}\left|c_{k 0}\right|^{2}\right. \\
& \left.+\sum_{k=-\infty}^{\infty} \sum_{n=1}^{\infty}\left(k^{2}+n^{4}\right)\left|c_{k n}\right|^{2}\right\}^{1 / 2}
\end{aligned}
$$

for $u=\sum_{k=-\infty}^{\infty} \sum_{n=0}^{\infty} c_{k n} e^{i k t} \cos n x \in \mathscr{X}$. We set $X:=\mathscr{X}^{4}$. We denote by $\mathscr{V}$ (resp., $\mathscr{W}$ ) the subspace of $\mathscr{X}$ (resp., $\mathscr{Y}$ ) consisting of steady functions, that is,

$$
\begin{gathered}
\mathscr{V}:=\left\{\sum_{n=0}^{\infty} c_{n} \cos n x ; c_{n} \in \mathbb{R} \text { and } \sum_{n=0}^{\infty} n^{4} c_{n}^{2}<\infty\right\}, \\
\mathscr{W}:=L^{2}(0, \pi) .
\end{gathered}
$$

We set $X_{1}:=\mathscr{V}^{4}, Y_{1}:=\mathscr{W}^{4}, X_{2}:=X_{1}^{\perp}$, and $Y_{2}:=Y_{1}^{\perp}$.

We set $\Lambda:=(\lambda, \sigma)$. We define $g(\Lambda, u):=u_{t}-\sigma \varphi(\lambda, u)$ and $l:=\left(l_{1}, l_{2}\right): X \rightarrow \mathbb{R}^{2}$ by

$$
\begin{gathered}
l_{1} u:=\frac{1}{\pi^{2}}\left(u_{1}, \sin t\right)_{y} \\
l_{2} u:=\frac{1}{\pi^{2}}\left(u_{1}, \cos t\right)_{y} \text { for } u=\left(u_{1}, u_{2}, u_{3}, u_{4}\right) \in X .
\end{gathered}
$$

Namely, $l_{1} u$ (resp., $l_{2} u$ ) equals to the Fourier coefficient of $\sin t$ (resp., cost) for $u_{1}$. Let $J: \mathbb{R}^{2} \times X \rightarrow \mathbb{R}^{2} \times Y$ be defined by (4) with $m=2$. We define $\widehat{J}: \mathbb{R}^{2} \times X \rightarrow \mathbb{R}^{2} \times Y$ by

$$
\begin{aligned}
\widehat{J}\left(\begin{array}{c}
\Lambda \\
v \\
w
\end{array}\right):= & \left(\begin{array}{c}
l w-\mathbf{e}_{1} \\
\varphi(\lambda, v) \\
g_{u}(\Lambda, v) w
\end{array}\right) \in \mathbb{R}^{2} \times Y_{1} \times Y_{2} \\
& \text { for }(\Lambda, v, w) \in \mathbb{R}^{2} \times X_{1} \times X_{2} .
\end{aligned}
$$

Then, (H1) is equivalent to the following (K1) since $\sigma_{0} \neq 0$ and $\varphi\left(\lambda_{0}, v_{0}\right)=0$ :

(K1) A point $(\Lambda, v, w)=\left(\Lambda_{0}, v_{0}, w_{0}\right)$ is an isolated solution of the extended system $\widehat{J}(\Lambda, v, w)=0$.

Similarly, (H2) is equivalent to the following (K2):

(K2) The linear operator $\left.\varphi_{u}\left(\lambda_{0}, v_{0}\right)\right|_{X_{1}}: X_{1} \rightarrow Y_{1}$ is bijective. 
4.3. A Verification Result. By our numerical verification method described in Section 4.5, we verified the existence of a Hopf bifurcation point $\left(\lambda_{0}, v_{0}\right)$ of (B) satisfying

$$
\begin{aligned}
& \left|\lambda_{0}-\tilde{\lambda}_{0}\right|^{2}+\left|\sigma_{0}-\widetilde{\sigma}_{0}\right|^{2}+\left\|v_{0}-\widetilde{v}_{0}\right\|_{X}^{2}+\left\|w_{0}-\widetilde{w}_{0}\right\|_{X}^{2} \\
& \quad \leq\left(6.46 \times 10^{-8}\right)^{2} .
\end{aligned}
$$

Here, $\left(\left(\lambda_{0}, \sigma_{0}\right), v_{0}, w_{0}\right)$ is an isolated solution of the extended system $\widehat{J}(\Lambda, v, w)=0$, and $\left(\left(\widetilde{\lambda}_{0}, \widetilde{\sigma}_{0}\right), \widetilde{v}_{0}, \widetilde{w}_{0}\right)$ is its numerical approximation. The initial period of bifurcating periodic solutions is $2 \pi \sigma_{0}$. We have $w_{0} \in W_{1}$, which is defined by (42) below. We set $\tilde{\lambda}_{0}:=0.9541600594715$ and $\widetilde{\sigma}_{0}:=$ 0.4534602327315 . The functions $\widetilde{v}_{0}$ and $\widetilde{w}_{0}$ have the forms

$$
\begin{aligned}
& \widetilde{v}_{0}:=\mathbf{a}+\mathbf{b} \cos x+\mathbf{c} \cos 2 x \quad \text { with } \mathbf{a}, \mathbf{b}, \mathbf{c} \in \mathbb{Q}^{4}, \\
& \widetilde{w}_{0}:=\left(\mathbf{p}_{1} \sin t+\mathbf{p}_{2} \cos t\right)+\left(\mathbf{q}_{1} \sin t+\mathbf{q}_{2} \cos t\right) \cos x \\
&+\left(\mathbf{r}_{1} \sin t+\mathbf{r}_{2} \cos t\right) \cos 2 x
\end{aligned}
$$

with $\mathbf{p}_{k}, \mathbf{q}_{k}, \mathbf{r}_{k} \in \mathbb{Q}^{4}(k=1,2)$. We have

$$
\begin{aligned}
& \left\|\widetilde{v}_{0}\right\|_{X} \in(20.8,20.9), \quad\left\|\widetilde{w}_{0}\right\|_{X} \in(4.25,4.26), \\
& \left\|\widehat{J}\left(\widetilde{\Lambda}_{0}, \widetilde{v}_{0}, \widetilde{w}_{0}\right)\right\|_{\mathbb{R}^{2} \times Y} \in\left(4.57 \times 10^{-9}, 4.58 \times 10^{-9}\right) .
\end{aligned}
$$

Here, $\widetilde{\Lambda}_{0}:=\left(\widetilde{\lambda}_{0}, \widetilde{\sigma}_{0}\right)$.

Remark 5. In the process of deriving the above result, we often used computer arithmetic with double precision, without taking into account the effects of the round-off errors, though (31) holds rigorously. It is sufficient, however, for our purpose, which is to check that Theorem 1 is applicable to the numerical verification method. We can make the above result completely rigorous if we always use the interval arithmetic or the computational method described in [2, Section 2.1] in the process of deriving the conclusion.

4.4. Principle of Our Numerical Verification Method. In this subsection, we present two important abstract results in our numerical verification method.

Let $\mathscr{H}$ and $\mathscr{K}$ be Banach spaces in what follows in this subsection. We assume $F \in C^{1}(\mathscr{H}, \mathscr{K})$. We can immediately obtain the following result from [8, Theorem 3.2].

Theorem 6 (the convergence theorem of simplified Newton method). Let $\tilde{u}_{0} \in \mathscr{H}$ and $A:=F^{\prime}\left(\tilde{u}_{0}\right)$ be bijective. We define a map $G: \mathscr{H} \rightarrow \mathscr{H}$ by

$$
G(u):=u-A^{-1} F(u) .
$$

Let $R>0$ be a constant and $b:[0, R] \rightarrow[0, \infty)$ be a nondecreasing function such that

$$
\sup \left\{\left\|G^{\prime}(u)\right\| ; u \in \overline{B\left(\widetilde{u}_{0} ; r\right)}\right\} \leq b(r) \quad \text { for any } r \in[0, R] \text {. }
$$

Let $\varepsilon_{0} \geq 0$ be a constant such that

$$
\left\|A^{-1} F\left(\tilde{u}_{0}\right)\right\| \leq \varepsilon_{0} .
$$

We assume that there exist constants $r_{0}$ and $r_{1}$ such that $0<r_{0} \leq r_{1} \leq R$

$$
\varepsilon_{0}+\int_{0}^{r_{0}} b(r) d r \leq r_{0} \text { and } b\left(r_{1}\right)<1 .
$$

Then, the equation $F(u)=0$ has an isolated solution $u_{0} \in$ $\overline{B\left(\tilde{u}_{0} ; r_{0}\right)}$. Moreover, the solution of $F(u)=0$ is unique in $\overline{B\left(\widetilde{u}_{0} ; r_{1}\right)}$.

Theorem 6 plays a central role in our numerical verification method.

Remark 7. Theorem 6 is a corollary of [9, Theorem 1.1]. Though the latter is in general stronger than the former, the former is simpler. The former works well for our present problem since the residue of the approximate solution is very small (see (31)).

In applying Theorem 6 , it is very important to find an upper bound of the norm of $A^{-1}$. Proposition 8 below is useful with respect to this point. We also need it in order to check the condition (H2).

Let $\mathscr{H}_{0}$ and $\mathscr{H}_{c}$ be closed subspaces of $\mathscr{H}$ such that $\mathscr{H}=$ $\mathscr{H}_{0} \oplus \mathscr{H}_{c}$. Let $\mathscr{K}_{0}$ and $\mathscr{K}_{c}$ be closed subspaces of $\mathscr{K}$ such that $\mathscr{K}=\mathscr{K}_{0} \oplus \mathscr{K}_{c}$. We denote by $P_{j} \in \mathscr{L}(\mathscr{H})$ the projection defined by $P_{j} u:=u_{j}$ for $u=u_{0}+u_{c} \in \mathscr{H}$ with $u_{j} \in \mathscr{H}_{j}(j=$ $0, c)$. We denote by $Q_{j} \in \mathscr{L}(K)$ the projection defined by $Q_{j} v:=v_{j}$ for $v=v_{0}+v_{c} \in \mathscr{K}$ with $v_{j} \in \mathscr{K}_{j}(j=0, c)$. Let $T \in \mathscr{L}(\mathscr{H}, \mathscr{K})$ and $\mathscr{L}: \mathscr{H} \rightarrow \mathscr{K}$ be a closed linear operator. We set $A:=L+T$. We assume

$$
\mathscr{R}\left(P_{0}\right) \subset \mathscr{D}(L), \quad L P_{0}=Q_{0} L \text { on } \mathscr{D}(L) .
$$

We define $A_{0}:=L+Q_{0} T P_{0}: \mathscr{H} \rightarrow \mathscr{K}$. We denote by $I_{\mathscr{H}}$ (resp., $I_{\mathscr{K}}$ ) the identity operator on $\mathscr{H}$ (resp., $\mathscr{K}$ ) and simply by $I$ the operators $I_{\mathscr{H}}$ and $I_{\mathscr{K}}$ when there is no ambiguity.

Proposition 8. In addition to the above assumptions, we assume that $A_{0}$ is bijective and that $S:=A_{0}^{-1} Q_{c} T(I-$ $\left.A_{0}^{-1} P_{0} T\right) P_{c} \in \mathscr{L}(H)$ satisfies $\|S\|<1$. Then, $A$ is bijective. Moreover, we have

$$
\begin{aligned}
& P_{0} A^{-1} Q_{0} \\
& =A_{0}^{-1}\left\{Q_{0}+Q_{0} T P_{c}(I+S)^{-1} A_{0}^{-1} Q_{c} T A_{0}^{-1} Q_{0}\right\}\left(:=A^{11}\right), \\
& P_{0} A^{-1} Q_{c}=A_{0}^{-1} Q_{0} T P_{c}(I+S)^{-1} A_{0}^{-1} Q_{c}\left(:=A^{12}\right), \\
& P_{c} A^{-1} Q_{0}=-(I+S)^{-1} A_{0}^{-1} Q_{c} T Q_{0} A_{0}^{-1} Q_{0}\left(:=A^{21}\right), \\
& P_{c} A^{-1} Q_{c}=(I+S)^{-1} A_{0}^{-1} Q_{c}\left(:=A^{22}\right) .
\end{aligned}
$$

Proposition 8 is a generalization of [9, Proposition 2.1] and can be proved in the same way. We note that we can apply Proposition 8 to our problem but cannot directly apply [9, Proposition 2.1].

Finally, we describe how Proposition 8 works well for our problem. In our present context, we can assume in addition the following (A1)-(A3): 
(A1) $\mathscr{H}$ and $\mathscr{K}$ are Hilbert spaces, and $\mathscr{H}$ is embedded continuously and densely in $\mathscr{K}$,

(A2) there is a CONS $\left\{\phi_{n}\right\}_{n=1}^{\infty}$ of $\mathscr{H}$ such that $\phi_{n}$ is an eigenfunction of $L$ for any $n \in \mathbb{N}$.

Let $\mathscr{H}_{n}$ and $\mathscr{K}_{n}$ be subspaces of $\mathscr{H}$ and $\mathscr{K}$, respectively, such that both $\mathscr{H}_{n}$ and $\mathscr{K}_{n}$ are equal to $\operatorname{span}\left\{\phi_{j}\right\}_{j=1}^{n}$ as sets. Let $P_{n}$ (resp., $Q_{n}$ ) be the orthogonal projection on $\mathscr{H}$ (resp., $\mathscr{K}$ ) onto $\mathscr{H}_{n}\left(\right.$ resp., $\left.\mathscr{K}_{n}\right)$,

(A3) $\left\|T\left(I-P_{n}\right)\right\|_{\mathscr{H} \rightarrow \mathscr{K}} \rightarrow 0$ and $\left\|\left(I-Q_{n}\right) T\right\|_{\mathscr{H} \rightarrow \mathscr{K}} \rightarrow$ 0 as $n \rightarrow \infty$.

We show that Proposition 8 works well if we take $\mathscr{H}_{0}:=\mathscr{H}_{n}$ and $\mathscr{K}_{0}:=\mathscr{K}_{n}$ for sufficiently large $n$. Let $A_{n}:=L+Q_{n} T P_{n}$ : $\mathscr{H} \rightarrow \mathscr{K}$.

Proposition 9. Under the assumptions above we have the following.

(i) If $A$ is bijective, then $A_{n}$ is bijective for sufficiently large $n \in \mathbb{N}$, and we have

$$
A_{n}^{-1} \longrightarrow A^{-1} \text { in } \mathscr{L}(\mathscr{K}, \mathscr{H}) \text { as } n \longrightarrow \infty \text {. }
$$

(ii) If $A_{n}$ is bijective and

$$
\left\|A_{n}^{-1}\right\|\left\|T-Q_{n} T P_{n}\right\|<1,
$$

then $A$ is bijective with the estimate $\left\|A^{-1}\right\| \leq$ $\left\|A_{n}^{-1}\right\| /\left(1-\left\|A_{n}^{-1}\right\|\left\|T-Q_{n} T P_{n}\right\|\right)$.

(iii) We have

$$
\left\|A_{n}^{-1}\right\| \longrightarrow\left\|A^{-1}\right\| \quad \text { as } n \longrightarrow \infty
$$

Here, we formally define $\left\|A_{n}^{-1}\right\|:=\infty$ (resp., $\left.\left\|A^{-1}\right\|:=\infty\right)$ when $A_{n}$ (resp., $\left.A\right)$ is not bijective.

Proof. We set $B_{n}:=T-Q_{n} T P_{n} \in \mathscr{L}(\mathscr{H}, \mathscr{K})$. Then, $A-A_{n}=$ $B_{n}$ on $\mathscr{D}(L)$. We obtain from (A3) that $\left\|B_{n}\right\| \rightarrow 0$ as $n \rightarrow \infty$.

(i) Let $A$ be bijective. Then, $\left\|A^{-1}\right\|\left\|B_{n}\right\|<1$ for sufficiently large $n$. Therefore, by [2, Corollary 2.4.1], $A_{n}$ is bijective for sufficiently large $n$, and we obtain (38).

(ii) We immediately obtain the desired conclusion from [2, Corollary 2.4.1].

(iii) If $A$ is bijective, (i) implies (40). So, we consider the case where $A$ is not bijective. We proceed by contradiction. We assume that $\lim _{n \rightarrow \infty}\left\|A_{n}^{-1}\right\| \neq \infty$. Then, $\liminf _{n \rightarrow \infty}\left\|A_{n}^{-1}\right\|<\infty$. So, there exists a large number $N \in \mathbb{N}$ such that $\left\|A_{N}^{-1}\right\|\left\|B_{N}\right\|<1$. So, (ii) implies that $A$ is bijective. This contradicts to our assumption. Therefore, (40) holds.

We consider the case where the condition of Proposition 8 is satisfied. Let $u \in \mathscr{K}$. The estimate (41) below is useful when we apply Theorem 6 to concrete problems. We denote by $A_{n}^{i j}$ the operator $A^{i j}$ with $A_{0}, P_{0}$,
$P_{c}, Q_{0}$, and $Q_{c}$ replaced by $A_{n}, P_{n}, I-P_{n}, Q_{n}$, and $I-Q_{n}$, respectively $(i, j=1,2)$. Then, we easily verify that

$$
\begin{aligned}
& \left\|A^{-1} u\right\| \\
& \quad \leq\left\{\left(\left\|A_{n}^{11} u\right\|+\left\|A_{n}^{12} u\right\|\right)^{2}+\left(\left\|A_{n}^{21} u\right\|+\left\|A_{n}^{22} u\right\|\right)^{2}\right\}^{1 / 2}
\end{aligned}
$$

and that the right-hand side of (41) converges to $\left\|A^{-1} u\right\|$ as $n \rightarrow \infty$ in view of (A3). So, the right-hand side of (41) is a sharp upper bounds of $\left\|A^{-1} u\right\|$ when $n$ is sufficiently large.

Remark 10. We denote by $S_{n}$ the operator $S$ defined in Proposition 8 with $A_{0}, P_{0}, P_{c}$, and $Q_{c}$ replaced by $A_{n}, P_{n}, I-P_{n}$ and $I-Q_{n}$, respectively. Though (39) is a sufficient condition for the existence of $A^{-1}$, another sufficient condition $\left\|S_{n}\right\|<1$ is in general more efficient. The reason is that $\left\|T\left(I-P_{n}\right)\right\|$ converges to $0(n \rightarrow \infty)$ more rapidly in general than $\left\|\left(I-Q_{n}\right) T\right\|$.

4.5. Derivation of Our Verification Result. Here, we describe the outline of derivation of our verification result in Section 4.3. We define closed subspaces of $X_{2}$ :

$$
\begin{gathered}
W_{1}:=\left\{\sum_{n=0}^{\infty}\left(\mathbf{a}_{n} \cos t+\mathbf{b}_{n} \sin t\right) \cos n x \in X ; \mathbf{a}_{n}, \mathbf{b}_{n} \in \mathbb{R}^{4}\right\}, \\
W_{2}:=\left\{\sum_{|k| \geq 2} \sum_{n=0}^{\infty} \mathbf{c}_{k n} e^{i k t} \cos n x \in X ; \mathbf{c}_{k n} \in \mathbb{C}^{4}\right\} .
\end{gathered}
$$

We similarly define closed subspaces of $Y_{2}$ :

$$
\begin{gathered}
Z_{1}:=\left\{\sum_{n=0}^{\infty}\left(\mathbf{a}_{n} \cos t+\mathbf{b}_{n} \sin t\right) \cos n x \in Y ; \mathbf{a}_{n}, \mathbf{b}_{n} \in \mathbb{R}^{4}\right\}, \\
Z_{2}:=\left\{\sum_{|k| \geq 2} \sum_{n=0}^{\infty} \mathbf{c}_{k n} e^{i k t} \cos n x \in Y ; \mathbf{c}_{k n} \in \mathbb{C}^{4}\right\} .
\end{gathered}
$$

Clearly, $X_{2}=W_{1} \oplus W_{2}$, and $Y_{2}=Z_{1} \oplus Z_{2}$. We set $J_{*}:=$ $\left.\widehat{J}\right|_{\mathbb{R}^{2} \oplus X_{1} \oplus W_{1}}: \mathbb{R}^{2} \oplus X_{1} \oplus W_{1} \rightarrow \mathbb{R}^{2} \oplus Y_{1} \oplus Z_{1}$ and $K:=$ $\left.\widehat{J}\right|_{W_{2}}=\left.g_{u}^{0}\right|_{W_{2}}: W_{2} \rightarrow Z_{2}$. Here, we replace the symbol of direct product by the direct sum (see the notation (8) just after Section 1), which helps us to describe the decomposition of $\widehat{J}$. Then, we can represent $\widehat{J}$ by the direct sum of maps: $\widehat{J}=J_{*} \oplus K$. Since $w_{0} \in W_{1}$, the condition (K1) is equivalent to the following (L1) and (L2):

(L1) A point $(\Lambda, v, w)=\left(\Lambda_{0}, v_{0}, w_{0}\right)$ is an isolated solution of the extended system $J_{*}(\Lambda, v, w)=0$.

(L2) The linear operator $K: W_{2} \rightarrow Z_{2}$ is bijective.

By the above discussion and Theorem 1 , it suffices to verify (L1), (L2), and (H2) in order to show that $(\lambda, v)=\left(\lambda_{0}, v_{0}\right)$ is a Hopf bifurcation point of (B). We verified (L1) and (29) by applying Theorem 6 with $F:=J_{*}$ and $\widetilde{u}_{0}:=$ $\left(\widetilde{\Lambda}_{0}, \widetilde{v}_{0}, \widetilde{w}_{0}\right)$, and (L2), (H2) by Proposition 8 . We need the 
following embedding inequality to check the conditions in Theorem 6 and Proposition 9. For simplicity, we denote $\|\cdot\|_{p}:=\|\cdot\|_{L^{p}(D)}$.

Proposition 11. We have

$$
\|u\|_{6} \leq C_{6}\|u\|_{x} \quad \text { for any } u \in \mathscr{X}
$$

where $C_{6}:=\{(1 / 2)(1 / \pi+2)(1 / \pi+3)\}^{1 / 3} \in(1.566,1.567)$.

Proof. The proof is similar to that of [2, Proposition 2.3.1].

First, let $-\infty<a<b<\infty$. In a similar way to the proof of $[2$, Lemma 2.3.1(ii)], we easily verify that

$$
\begin{aligned}
& \max _{x \in[a, b]}|f(x)| \\
& \leq \int_{a}^{b}\left|f^{\prime}(x)\right| d x \\
& \quad+\frac{1}{b-a} \int_{a}^{b}|f(x)| d x \text { for any } f \in H^{1}(a, b) .
\end{aligned}
$$

We easily verify that

$$
\max \left(\|u\|_{2},\left\|u_{x}\right\|_{2},\left\|u_{t}\right\|_{2}\right) \leq\|u\|_{x} \quad \text { for any } u \in \mathscr{X} .
$$

We set $\mathscr{X}_{0}:=\left\{\sum_{k=-M}^{M} \sum_{n=0}^{N} c_{k n} e^{i k t} \cos n x \in \mathscr{X} ; c_{k n} \in \mathbb{C}\right.$ and $M, N \in \mathbb{N} \cup\{0\}\}$. It suffices to prove (44) for any $u \in \mathscr{X}_{0}$ since $\mathscr{X}_{0}$ is dense in $\mathscr{X}$. Let $u \in \mathscr{X}_{0}$. It follows from (45) and [2, Lemma 2.3.1(ii)] that

$$
\begin{aligned}
& \int_{0}^{2 \pi} d t \int_{0}^{\pi} u^{2} d x \\
& \quad \leq \int_{0}^{2 \pi} \max _{y \in[0, \pi]}|u(t, y)| d t \int_{0}^{\pi} \max _{\tau \in[0,2 \pi]}|u(\tau, x)| d x \\
& \quad \leq\left(\frac{1}{\pi}\|u\|_{1}+\left\|u_{x}\right\|_{1}\right)\left(\frac{1}{2 \pi}\|u\|_{1}+\frac{1}{2}\left\|u_{t}\right\|_{1}\right) .
\end{aligned}
$$

Let $v \in \mathscr{X}_{0}$. We substitute $u=v^{j}(j=2,3)$ to (47). Then, we obtain from (46) and Schwarz inequality that

$$
\begin{aligned}
\|v\|_{4}^{4} & \leq\left(\frac{1}{\pi}\|v\|_{2}^{2}+2\left\|v v_{x}\right\|_{1}\right)\left(\frac{1}{2 \pi}\|v\|_{2}^{2}+\left\|v v_{t}\right\|_{1}\right) \\
& \leq\left(\frac{1}{\pi}\|v\|_{2}^{2}+2\|v\|_{2}\left\|v_{x}\right\|_{2}\right)\left(\frac{1}{2 \pi}\|v\|_{2}^{2}+\|v\|_{2}\left\|v_{t}\right\|_{2}\right) \\
& \leq \frac{1}{2}\left(\frac{1}{\pi}+2\right)^{2}\|v\|_{x}^{4}, \\
\|v\|_{6}^{6} & \leq\left(\frac{1}{\pi}\|v\|_{3}^{3}+3\left\|v^{2} v_{x}\right\|_{1}\right)\left(\frac{1}{2 \pi}\|v\|_{3}^{3}+\frac{3}{2}\left\|v^{2} v_{t}\right\|_{1}\right) \\
& \leq\|v\|_{4}^{4}\left(\frac{1}{\pi}\|v\|_{2}+3\left\|v_{x}\right\|_{2}\right)\left(\frac{1}{2 \pi}\|v\|_{2}+\frac{3}{2}\left\|v_{t}\right\|_{2}\right) \\
& \leq\|v\|_{4}^{4} \cdot \frac{1}{2}\left(\frac{1}{\pi}+3\right)^{2}\|v\|_{x}^{2} .
\end{aligned}
$$

By (48) and (49), we have (44) with $u$ replaced by $v$. The proof is complete.
Remark 12. Though our numerical verification method is not difficult, the verification process consists of a lot of steps and is very complicated. So, we omit its details here. They are much similar to the discussions in $[2,9]$. In particular, the verification process is described fully in detail in [2].

\section{Final Remarks}

Remark 13. Our main result Theorem 1 is new and useful from the practical and applied mathematical point of view. It does not mean, however, that it is new from the theoretical point of view, though there is no known result equivalent to our main result with $m \geq 2$ as far as the author knows. In view of Proposition 4, Theorem 1 is theoretically considered as a corollary of [5, Theorem 1.7] for the case $m=1$ and is generalization of it for the case $m \geq 2$. We refer to [10] by Hale and [11] by López-Gómez where some related bifurcation theorems were obtained. They are generalization of [5, Theorem 1.7] different from our main result. Actually, Theorem 1 implies Theorem 2, as shown in our work mentioned in Remark 14 below. On the other hand, the bifurcation theorems in $[10,11]$ do not imply Theorem 2.

Remark 14. In a near future work, we will study the equivalent relations between conditions in Theorem 1 with $m=2$ and those in Theorem 2 and show that Theorem 1 with $m=2$ is stronger than the existence part of [12, Theorem 1.11].

Remark 15. Nishida et al. studied problems of stability and bifurcation of solutions for some fluid equations in $[13,14]$. We can apply the same technique as in $[13,14]$ to check rigorously the condition (C3) in Theorem 2 by the numerical method.

Let $A:=\sigma_{0} f_{y}\left(\lambda_{0}, y_{0}\right), \psi_{0} \in N(A-i)-\{0\}$, and $\psi_{*} \epsilon$ $N\left(A^{*}+i\right)-\{0\}$. Then, we can obtain in the same way as in [13] and [14, Section 4] that

$$
\operatorname{Re} \mu^{\prime}\left(\lambda_{0}\right)=\operatorname{Re} \frac{\left(f_{\lambda y}^{0} \psi_{0}+f_{y y}^{0} \nu_{*} \psi_{0}, \psi_{*}\right)}{\left(\psi_{0}, \psi_{*}\right)}
$$

Here, $f_{\lambda y}^{0}:=f_{\lambda y}\left(\lambda_{0}, y_{0}\right), f_{y y}^{0}:=f_{y y}\left(\lambda_{0}, y_{0}\right)$, and $v_{*} \in \mathbb{R}^{d}$ satisfies $f_{\lambda}\left(\lambda_{0}, y_{0}\right)+f_{y}\left(\lambda_{0}, y_{0}\right) v_{*}=0$. We can check the condition (C3) since we can find by the numerical verification method an accurate approximate value of the right-hand side of (50) with a rigorous error bound.

\section{Acknowledgments}

The author would like to express his sincere gratitude to Professor Atsushi Yagi and Professor Takaaki Nishida for their encouragement. The author is grateful to the referees for constructive comments.

\section{References}

[1] T. Kawanago, "A symmetry-breaking bifurcation theorem and some related theoremsapplicable to maps having unbounded derivatives," Japan Journal of Industrial and Applied Mathematics, vol. 21 , no. 1 , pp. 57-74, 2004, Corrigendum to this paper: 
Japan Journal of Industrial and Applied Mathematics, vol. 22, pp. 147, 2005.

[2] T. Kawanago, "Computer assisted proof to symmetry-breaking bifurcation phenomena in nonlinear vibration," Japan Journal of Industrial and Applied Mathematics, vol. 21, no. 1, pp. 75-108, 2004.

[3] Y. Watanabe and M. T. Nakao, "Numerical verification method of solutions for elliptic equations and its application to the Rayleigh-Bénard problem," Japan Journal of Industrial and Applied Mathematics, vol. 26, no. 2-3, pp. 443-463, 2009.

[4] M. T. Nakao, Y. Watanabe, N. Yamamoto, T. Nishida, and M.N. Kim, "Computer assisted proofs of bifurcating solutions for nonlinear heat convection problems," Journal of Scientific Computing, vol. 43, no. 3, pp. 388-401, 2010.

[5] M. G. Crandall and P. H. Rabinowitz, "Bifurcation from simple eigenvalues," Functional Analysis, vol. 8, pp. 321-340, 1971.

[6] M. Reed and B. Simon, Functional analysis I, Academic Press, New York, NY, USA, 2nd edition, 1980.

[7] M. Kubíček and M. Holodniok, "Algorithms for determination of period-doubling bifurcation points in ordinary differential equations," Journal of Computational Physics, vol. 70, no. 1, pp. 203-217, 1987.

[8] T. Kawanago, "Error analysis of Galerkin's method for semilinear equations," Journal of Applied Mathematics, vol. 2012, Article ID 298640, 15 pages, 2012.

[9] T. Kawanago, "Improved convergence theorems of Newton's method designed for the numerical verification for solutions of differential equations," Journal of Computational and Applied Mathematics, vol. 199, no. 2, pp. 365-371, 2007.

[10] J. K. Hale, "Bifurcation from simple eigenvalues for several parameter families," Nonlinear Analysis, vol. 2, no. 4, pp. 491497, 1978.

[11] J. López-Gómez, "Multiparameter local bifurcation based on the linear part," Journal of Mathematical Analysis and Applications, vol. 138, no. 2, pp. 358-370, 1989.

[12] M. G. Crandall and P. H. Rabinowitz, "The Hopf bifurcation theorem in infinite dimensions," Archive for Rational Mechanics and Analysis, vol. 67, no. 1, pp. 53-72, 1977.

[13] T. Iohara, T. Nishida, Y. Teramoto, and H. Yoshihara, "Benard-marangoni convection with a deformable surface," Sūrikaisekikenkyūsho Kōkyūroku, no. 974, pp. 30-42, 1996.

[14] T. Nishida, Y. Teramoto, and H. Yoshihara, "Bifurcation problems for equations of fluid dynamics and computer aided proof," in Proceedings of the 2nd Japan-China Seminar on Numerical Mathematics, vol. 14 of Lecture Notes in Numerical and Applied Analysis, pp. 145-157, Kinokuniya, Tokyo, Japan, 1995, Advances in numerical mathematics. 


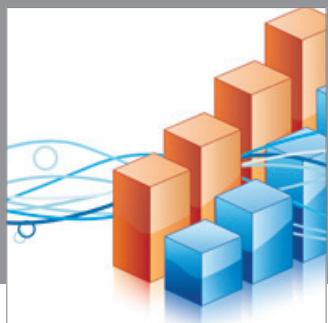

Advances in

Operations Research

mansans

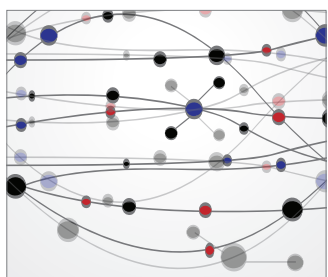

The Scientific World Journal
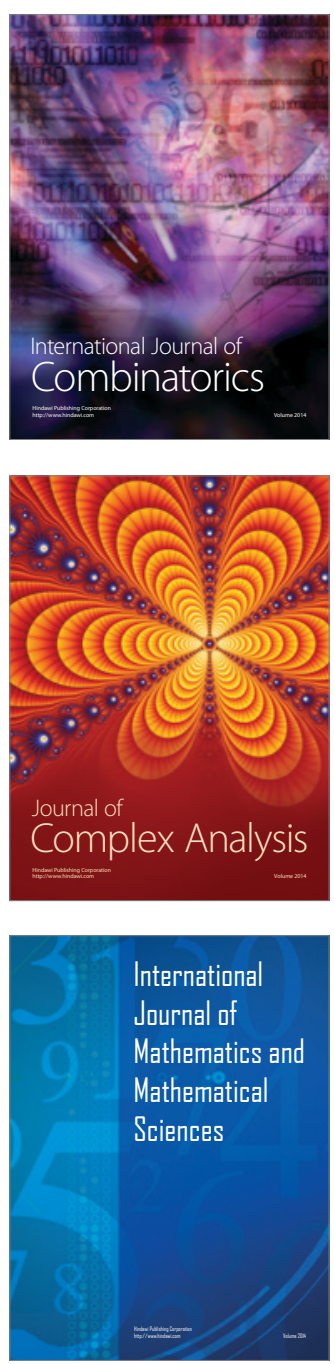
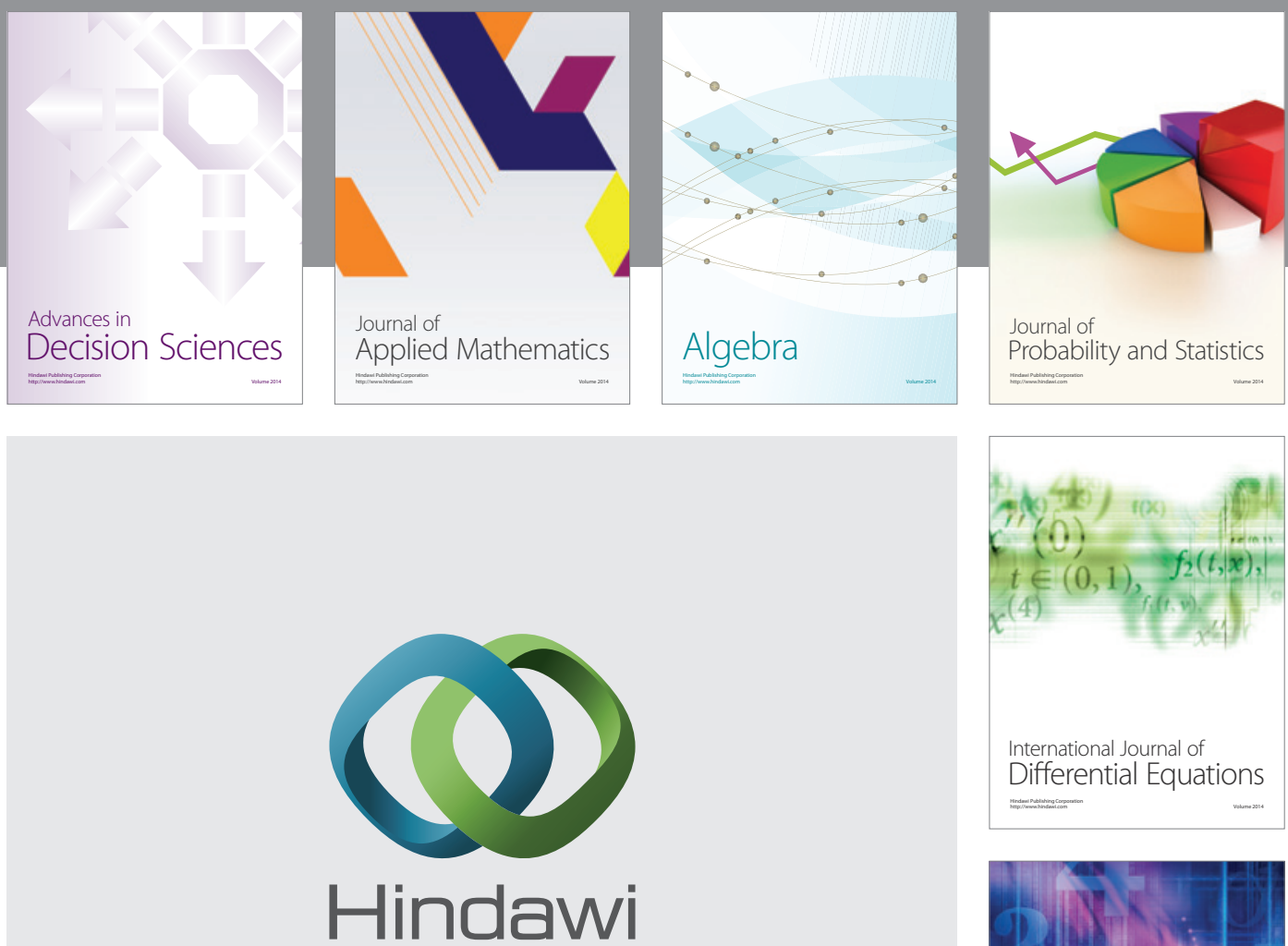

Submit your manuscripts at http://www.hindawi.com
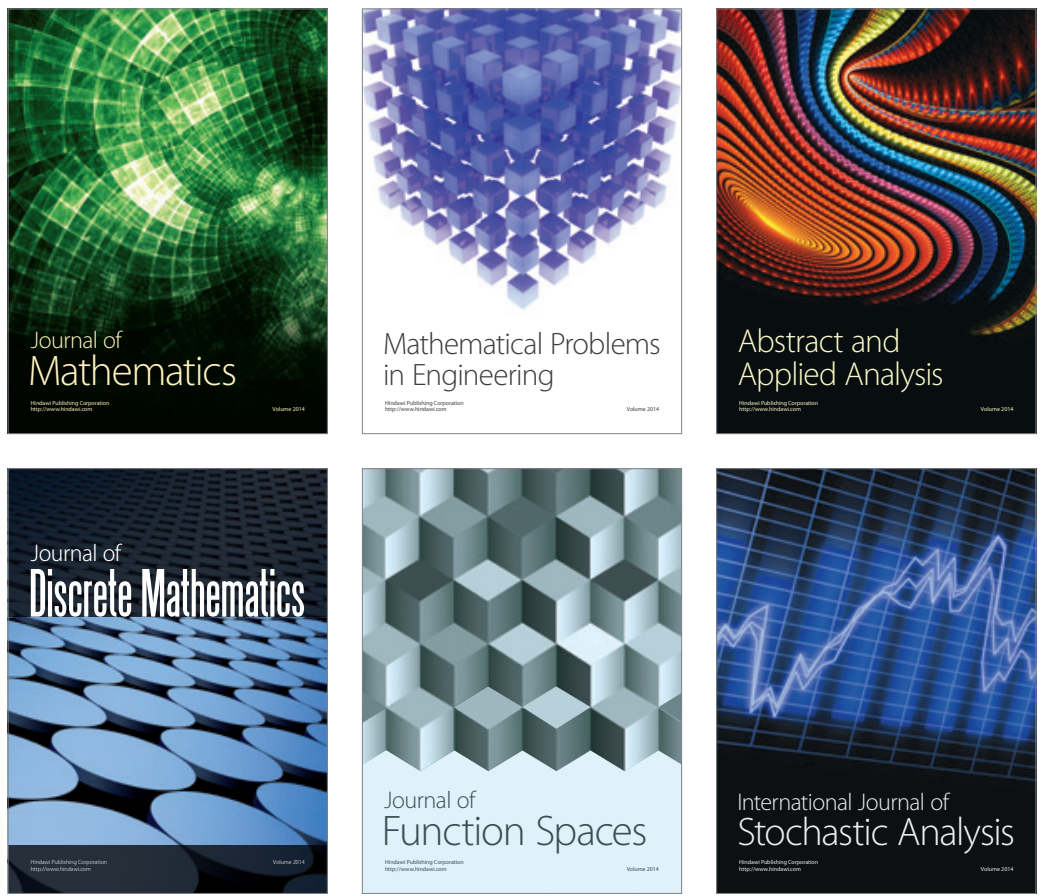

Journal of

Function Spaces

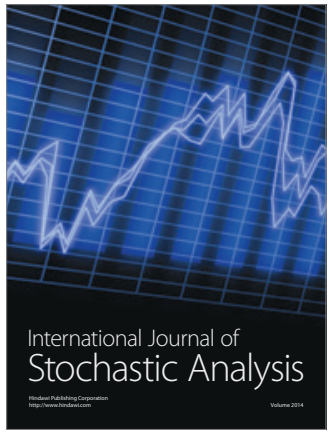

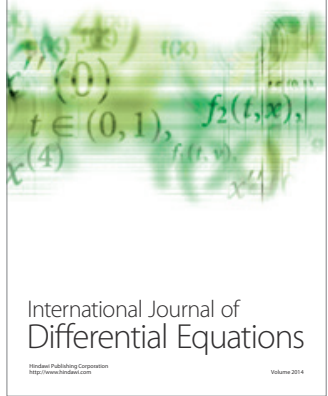
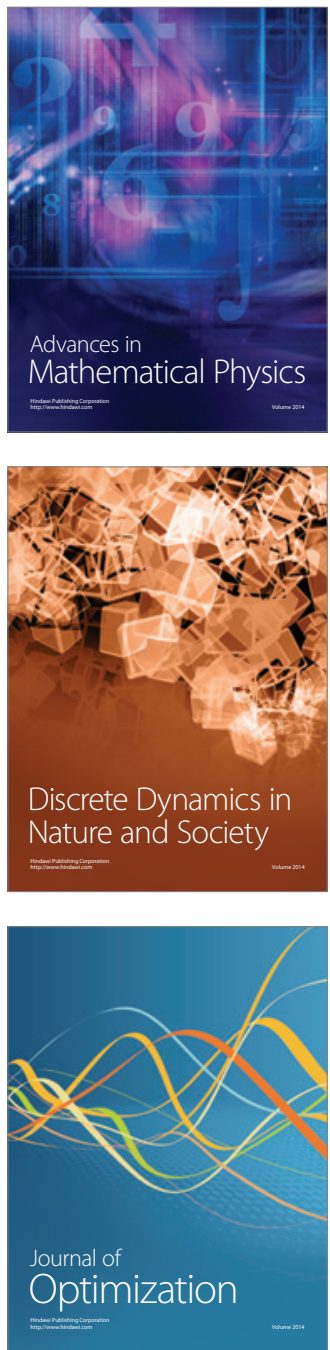